\title{
Paternal and maternal effects in a mosquito: A bridge for life history transition
}

\author{
Kylie Zirbel Yanchula ${ }^{1}$ and Barry Alto ${ }^{1}$ \\ ${ }^{1}$ University of Florida
}

April 28, 2020

\begin{abstract}
Parental (transgenerational) effects occur when the conditions experienced by a mother or father contribute to offspring phenotype. Here we show that parental larval diet in mosquitoes, Aedes aegypti, results in differential allocation of resources in offspring of parents depending on the nutritional condition (quality) of their mate. Maternal effects influenced the number of eggs produced by females as well as their lipid investment. Low nutrient females mated with high nutrient males laid eggs with significantly higher lipid content than those laid by high nutrient females. Paternal effects showed that when high nutrient males mated with low nutrient females, resulting eggs had higher lipid content than when low nutrient males mated with low nutrient females. Overall, our results are consistent with a pattern predicted by the differential allocation of resources hypothesis, when females experience nutritional deprivation, which asserts that mate quality directly influences reproductive allocation.
\end{abstract}

Running title: Paternal and maternal nutrition effects

Title: Paternal and maternal effects in a mosquito: A bridge for life history transition

List of Authors: 1. Kylie Zirbel Yanchula, Florida Medical Entomology Laboratory, Entomology and Nematology Department, Institute of Food and Agricultural Sciences, University of Florida, Vero Beach, FL 32962, USA. Email: kyanchula@martin.fl.us

2. Barry W. Alto, Florida Medical Entomology Laboratory, Entomology and Nematology Department, Institute of Food and Agricultural Sciences, University of Florida, Vero Beach, FL 32962, USA. Email: bwalto@ufl.edu.

Address Correspondence to:

Barry W. Alto

Florida Medical Entomology Laboratory

University of Florida

$2009^{\text {th }}$ Street S.E.

Vero Beach, FL 32962

Email: bwalto@ufl.edu

Tel. 772-266-6630

Fax. $772-778-7205$

Keywords : Mosquito, diet, cross-mating experiments, macronutrients, paternal effects, maternal effects Type of article : Letters 
Statement of authorship: KZY and BWA designed the study, KZY performed the research and collected data, KZY and BWA provided methods and materials, KZY and BWA performed statistical analyses, and KZY and BWA wrote the manuscript.

Data accessibility statement: We confirm that, should the manuscript be accepted, the data supporting the results will be archived in an appropriate public repository (Dryad) and the data DOI will be included at the end of the article.

Number of words : Abstract (144), Main text (4689), acknowledgements (62), references (2055), tables and figure legends (250), text boxes (0)

Number of references : 86

Number : Figures (2), tables (2), and text boxes (0)

\section{ABSTRACT}

Parental (transgenerational) effects occur when the conditions experienced by a mother or father contribute to offspring phenotype. Here we show that parental larval diet in mosquitoes, Aedes aegypti, results in differential allocation of resources in offspring of parents depending on the nutritional condition (quality) of their mate. Maternal effects influenced the number of eggs produced by females as well as their lipid investment. Low nutrient females mated with high nutrient males laid eggs with significantly higher lipid content than those laid by high nutrient females. Paternal effects showed that when high nutrient males mated with low nutrient females, resulting eggs had higher lipid content than when low nutrient males mated with low nutrient females. Overall, our results are consistent with a pattern predicted by the differential allocation of resources hypothesis, when females experience nutritional deprivation, which asserts that mate quality directly influences reproductive allocation.

\section{INTRODUCTION}

Transgenerational effects are considered a type of phenotypic plasticity inherited by offspring arising from the experiences of the parental generation (Carrière 1994).

These effects are important factors contributing to the ecology of organisms and can influence evolutionary responses to selection (Kirkpatrick and Lande 1989). The environmental conditions experienced by parents can enhance offspring fitness (Mousseau and Dingle 1991, Agrawal et al. 1999, Marshall and Uller 2007) or reduce offspring fitness (Vijendravarma et al. 2010). These effects can be maternally-derived and/or paternally derived, and are often context dependent (Qvarnström and Price 2001, Plaistow et al. 2006). While significantly more attention has been paid to transgenerational effects in organisms displaying parental care, these effects have been identified in a wide range of taxa including invertebrates (Mousseau and Fox 1998). In insects, mothers and fathers have been shown to influence different offspring traits, often in a sex-specific manner (e.g. Bonduriansky and Head 2007, Roth et al. 2009, Garcia-Gonzalez and Simmons 2011, Dew-Budd et al. 2016, Zirbel et al. 2018, Zirbel and Alto 2018), in part attributable to the evolution of sex-specific reproductive investment strategies (Zanchi et al. 2011).

The mosquito, Aedes aegypti, is one of the most important vectors of arthropod-borne (arbo) viruses including Zika, dengue, chikungunya, and yellow fever viruses. Previous studies on Ae. aegypti have characterized the influence of parental larval nutrition on offspring life history traits and mosquito-virus interactions (Zirbel et al. 2018, Zirbel and Alto 2018). These effects were maternally- and paternally-derived, as determined by experimental manipulations of parental mating crosses (Zirbel and Alto 2018). Despite the importance of these observations, no mechanisms were considered. While transgenerational effects have been widely documented for a variety of insects, the mechanisms behind these effects are largely speculative. Proposed mechanisms involved in transgenerational effects in insects include egg resource provisioning (Rossiter 1991, Sinervo 1991, Fox et al. 1999, Rolff 1999, McIntyre and Gooding 2000, Freitak et al. 2009, Gibbs et al. 2010, Urbanski et al. 2010 ), epigenetic marking (Bongiorni et al. 1999, Bonduriansky and Head 2007, Freitak et al. 2009, MacDonald 2012), sex-specific genomic imprinting (Bongiorni et al. 1999, MacDonald 2012), 
transfer of symbionts (Moran et al. 2008), transfer of immunodefence factors (Tidbury et al. 2010, Boots and Roberts 2012, Triggs and Knell 2012, Pölkki et al. 2012), and elevation of germline mutation (Bonduriansky and Head 2007). Of these, egg size is one of the more tractable ways to identify transgenerational effects and has not been investigated in this context for mosquitoes (Uller 2008).

Egg size is typically used as an indicator of reproductive investment among diverse organisms (Winkler and Wallin 1987, Sinervo 1990, Sinervo and Licht 1991, Bernardo 1996, Fox and Mousseau 1996, Fox et al. 1999, Giron and Casas 2003). Egg size variation has been documented in Ae. aegypti (Steinwascher 1984) and may be a proximate mechanism, along with nutrient provisioning, by which mothers can influence offspring phenotype. In a laboratory study, females from higher larval nutrient environments acquired larger amounts of lipid, glycogen, and proteins allowing them to provision a higher number of eggs compared to females in low food environments (Telang et al. 2006). How females allocate resources to eggs may influence offspring development and their fitness (Perez and Noriega 2012). Given that Ae. aegypti egg yolks are primarily composed of protein, lipids, glycogen, and water (Briegel et al. 2003), the quantities of these macronutrients may be differently allocated depending upon the nutritional status of the mother (Briegel 1990, Ziegler 1997) and possibly the nutritional status of the father. Further, limiting resources and developmental constraints lead to trade-offs between quantity and quality of offspring (eggs), a determinant of individual fitness and an influence on evolution of life histories (Stearns 1992).

During and after mating, female Ae. aegypti undergo many different behavioral and physiological changes from exposure to male ejaculate and seminal fluid proteins (sfps) produced by male accessory glands (Klowden and Chambers 1991, Sirot et al. 2011). These sfps may modulate use of proteins, hormone levels, and how sperm is utilized (Sirot et al. 2011) all of which may ultimately influence egg development generating nongenetic paternal effects (Simmons and Lovegrove 2019). Adult body size is associated with larval nutrition inAe. aegypti (Schneider et al. 2004). Small males likely experience seminal depletion more quickly than large males and semen depletion can influence female longevity and fecundity (Helinski and Harrington 2011). When mated with males depleted in sfps and or sperm, females produce fewer eggs and had reduced longevity relative to those that mated with non-depleted males (Helinski and Harrington 2011) and this may lead to paternal effects.

While experiments have considered the influence of female body size on egg size (Steinwascher 1984) and larval nutrition on the endocrinology of mosquito egg development (Telang et al. 2006), the influence of combined parental nutrition on egg size and nutrient composition has not been characterized. Previous research suggests that both maternal and paternal larval nutrition influences offspring life histories in Ae. aegypti (Zirbel et al. 2018, Zirbel and Alto 2018). Egg size is considered a tractable way to test for plasticity and parental effects (Uller 2008). Here we distinguish between maternal and paternal larval nutrition and mating cross influences on the number produced, average size, lipid content, and protein content of $A e$. aegyptieggs.

We use a mosquito model system to assess reproductive investment when mate quality varies by nutritional condition, to distinguish between differential allocation of resources and reproductive compensation hypotheses (Harris and Uller 2009). Mosquitoes are well-suited for testing these hypotheses since females are usually inseminated only once and retain sperm in a spermatheca to fertilize future ova (Fuchs et al. 1969), and so accurate assessment of the quality of potential mates may have lasting fitness consequences. Reproductive compensation predicts that parents increase reproductive effort when mating with low-quality mates to counteract negative effects of lower offspring fitness (i.e., reproductive compensation; increased investment with low-quality mates, Bluhm and Gowaty 2004, Gowaty et al. 2007). This hypothesis assumes that individuals are constrained to breed with individuals they do not prefer, and mate preferences predict variation in health and viability of offspring (Gowaty 2008). That is, parents increase reproductive investments in offspring to compensate for lowered offspring viability resulting from reproduction under constraints. In contrast, the differential allocation hypothesis asserts that mate quality directly influences reproductive effort/allocation (i.e., differential allocation; increased investment into offspring produced from high-quality mates, Burly et al. 1988, Gowaty et al. 2007). This work has achieved improved understanding of parental 
investment in mosquito offspring.

\title{
MATERIALS AND METHODS
}

\author{
Study Organism and Laboratory Colony
}

Ae. aegypti larvae were collected in 2012 from field containers in Key West, FL and used to establish a laboratory colony. Colony mosquitoes were reared in an insectary at $28 \pm 0.5^{\circ} \mathrm{C}$ with a $12: 12$ (light:dark) hour photoperiod. Mosquitoes were hatched in enamel pans $(24 \times 36 \times 5 \mathrm{~cm})$ containing $1.5 \mathrm{~L}$ of water and $200 \mathrm{mg}$ of larval food in cohorts of approximately 200. Larval food consisted of a 1:1 mixture of lactalbumin:Saccharomyces cerevisiae yeast which was provided twice a week in $200 \mathrm{mg}$ aliquots. Upon pupation, mosquitoes were transferred to water-filled cups and placed in plastic cages ( $45.7 \times 45.7 \times 45.7 \mathrm{~cm}$, BugDorm, MegaView Science Co. Ltd. Taichung, Taiwan) for emergence. Adults were sustained on a $10 \%$ sucrose solution provided in cotton wicks and females were given blood meals weekly from live chickens. Chicken care followed the animal use and care policies of the University of Florida's Institutional Animal Care and Use Committee (IACUC Protocol 201807682).

\section{Parental Treatment}

For this study, the $\mathrm{F}_{12}$ colony generation was used and will herein be referred to as the parental (P) generation. $\mathrm{P}$ generation larvae were hatched in enamel pans $(24 \times 36 \times 5 \mathrm{~cm})$ containing $1.5 \mathrm{~L}$ of water and $200 \mathrm{mg}$ of larval food. After 24 hours, P larvae were removed from nutrients and placed individually into cylindrical vials $(82 \times 26 \mathrm{~mm}$ ht. x dia.) containing $10 \mathrm{~mL}$ of water with either low $(0.75 \mathrm{mg})$ or high larval food $(3.0$ $\mathrm{mg}$ ). Low food larvae were hatched 1 week prior to high food larvae to ensure mating between $\mathrm{P}$ larvae treatment groups given that low food larvae take longer to develop. A total of 1400 high food and 1400 low food larvae were hatched and reared. Once mosquitoes emerged, adults were sexed and placed in treatment and sex specific cages in numbers no greater than 50 mosquitoes per cage. Adults were sustained on $10 \%$ sucrose solution. After all adults emerged, they were used in the following parental mating cross treatments:

High food $\mathrm{x}$ High food : HH High food $\mathrm{x}$ Low food : HL Low food $\mathrm{x}$ High food : LH Low food $\mathrm{x}$ Low food : LL

Mosquitoes were cold anesthetized and placed into treatment specific cages at a 1:1 male to female ratio for 7 days to allow for mating. In order to maintain the 1:1 male:female ratio, subsets of males were haphazardly selected from the high and low food treatments. After one week, females were allowed to blood feed on live chickens for one hour. After the feeding period, females were cold anesthetized and fully engorged blood-fed females were placed individually into cylindrical tubes $(82 \times 26 \mathrm{~mm}$ ht. $\mathrm{x}$ dia. $)$ containing a small piece of damp cotton and seed germination paper as an oviposition substrate. Females were given one week to oviposit after which egg papers and adult females were removed and stored at -80deg C until processed. Wings were removed from females and mounted onto slides for wing length determination using computer imaging software (i-Solution lit(c), AIC, Princeton, NJ). Wings were used to estimate body size (reviewed in Armbruster and Hutchinson 2002) and were measured in millimeters from alula to wing tip. See Figure 1 for experimental design and parental nutrient mating crosses.

Egg papers were photographed using an Olympus SZ61 stereomicroscope fitted with an Infinity 1 digital camera. Total egg number per female was recorded. Length and width measurements were taken for a subset of 10 eggs per female to approximate average egg size using the ImageJ 1.50i software. For females laying fewer than 10 eggs, all were measured. Under the assumption that egg shape is a prolate spheroid, egg volume was estimated using the methods described by Hawley (1985). Specifically, the following equation was used where $\mathrm{V}=$ volume, $\mathrm{L}=$ length, and $\mathrm{W}=$ width:

$\mathrm{V}=\pi \mathrm{LW}^{2} / 6$

Egg nutrient composition quantification assays 
Triacylglycerides were measured through colorimetric determination using the BioVision Triglyceride Quantification Kit (BioVision, Mountain View, CA; cat \#K622-100). Ten eggs per assay were manually homogenized using sterile pestles in centrifuge tubes with $1 \mathrm{~mL}$ of $5 \% \mathrm{NP}-40$ detergent (lysis buffer). Ten eggs were used to ensure measurements were within the range of sensitivity of the kit based upon previous studies on first instar larvae (Perez and Noriega 2012). We used the manufacturers protocol (BioVision) to perform the assay. Briefly, $30 \mu \mathrm{L}$ of homogenized egg sample were added to wells individually on a 96-well clear microplate, along with $46 \mu \mathrm{L}$ of triglyceride assay buffer, $2 \mu \mathrm{L}$ of triglyceride probe, and $2 \mu \mathrm{L}$ of triglyceride assay mix. Due to potential interference from endogenous compounds in the sample interfering with the assay, each sample well was intentionally spiked with $2 \mathrm{nmol}$ of triglyceride standard. Plates were mixed, covered with foil to protect from the light, and allowed to incubate at room temperature for 60 minutes. Plates were read on a spectrophotometer (Bio-Tek, Synergy HT) with absorbance set to $570 \mathrm{~nm}$. The assay kit contained serial dilutions of standards containing known quantities of lipids which were ran on a microplate in triplicate. Data produced from these standards was used to generate a standard curve which was used to quantify lipid content in the eggs.

Proteins were quantified using the Thermo Scientific Pierce $660 \mathrm{~nm}$ Protein Assay Kit (Pierce Biotechnology, Rockford, IL; cat \#22662). For protein quantification, 10 eggs were pooled and prepared for each assay to remain within the sensitivities of the kit as determined by studies on $1^{\text {st }}$ instar larvae (Perez and Noriega 2012). Eggs were prepared for protein analysis using the methods described by Giron et al. (2002). Specifically, 10 eggs were crushed using a sterile pestle into $800 \mu \mathrm{L}$ of physiological water containing $0.001 \%$ Triton X-100 (Pierce Biotechnology, Rockford, IL; cat \#85111) and placed at $4^{\circ} \mathrm{C}$ for five days to allow proteins to dissolve into the detergent for analysis. After five days, the protocol provided by Pierce Biotechnology for protein quantification was followed. Briefly, $8 \mu \mathrm{L}$ of sample and $150 \mu \mathrm{L}$ of protein assay reagent were added to each well of a 96-well clear microplate. Each sample was intentionally spiked with $2 \mu \mathrm{L}$ of $17 \%$ BSA standard to ensure that the assay would register within the BSA standard curve. Plates were mixed on a plate shaker set to medium speed for one minute and incubated at room temperature for 5 minutes. After incubation, plates were read at an absorbance of $660 \mathrm{~nm}$. Samples were quantified based upon a standard curve generated from standards.

\section{Statistical analyses}

Analysis of variance (ANOVA) was used to compare female size (wing length) among treatment groups (PROC GLM, SAS v. 9.3). Multivariate analysis of variance (MANOVA) was used to determine the contribution of parental larval nutrient mating cross effects on the quantity of eggs produced during the first gonotrophic cycle, egg volume, egg lipid content, and egg protein content (PROC GLM, SAS v. 9.3). Significant effects were further analyzed with multivariate pairwise contrasts of means adjusted using the sequential Bonferroni method $(\alpha=0.05)$. The relative contribution of each egg parameter to significant treatment effects and their relationship to each other (positive/negative) was measured using standardized canonical coefficients (Scheiner 2001). Specifically, to consider maternal contribution to egg number, volume, and composition eggs from HH vs. LH and HL vs. LL were compared. To consider paternal contribution to aforementioned effects HH vs. HL and LH vs. LL were compared. Lastly, to consider combined parental effects HH vs. LL and HL vs. LH were considered.

\section{RESULTS}

Of the 2,800 larvae, a total of 2,030 survived to adulthood (72.5\%). In the low food treatment, 689 males and 496 females emerged and in the high food treatment, 431 males and 414 females emerged. Of these, 207 males and 207 females were used for the HH treatment, 207 males and 207 females for the HL treatment, 248 males and 248 females for the LH treatment and 248 males and 248 females for the LL treatment. See Table 1 for summary statistics on parental treatment numbers, percent blood feeding, percent of blood fed females successfully laying eggs, and blood fed female wing length. When comparing high food females, ANOVA results showed that those that mated with high food males $(\mathrm{HH})$ did not have different mean wing lengths than those that mated with low food males (HL) $(p=0.9988)$. When comparing low food females, ANOVA 
results showed that those that mated with high food males (LH) did not have different mean wing lengths than those that mated with low food males (LL) $(p=0.7501)$. ANOVA also showed that high food blood fed females had significantly longer mean wing lengths than low food blood fed females (HH vs. LH, $p<0.0001$; HH vs. LL, $p<0.0001$; HL vs. LL, $p<0.0001$; HL vs. LH, $p<0.0001)$.

See Figure 2 for descriptive statistics on egg parameters measured. MANOVA showed significant parental nutrient mating cross effects on egg production (Pillai's trace $=0.444, p<0.0001$ ). Subsequent analyses demonstrated significant maternal nutrient effects on egg parameters measured (Table 2, HH vs. LH and HL vs. LL). In females that mated with high food males, egg count followed by lipid content contributed the most to this effect. Specifically, high food females that mated with high food males (HH) laid nearly 2 times more eggs than low food females that mated with high food males (LH). Low food females that mated with high food males laid eggs with 1.4 times higher lipid content than high food females that mated with high food males. In females that mated with low food males, egg count contributed the most to the effect. Specifically, high food females that mated with low food males (HL) laid 1.9 times more eggs than low food females that mated with low food males (LL).

Multivariate contrasts demonstrated significant paternal effects on egg production when males mated with low food females (LH vs. LL, $p=0.0113$ ) but not when males mated with high food females (HH vs. HL, $p=$ 0.4591). In males that mated with low food females, lipid content contributed most to the effect. Specifically, high food males that mated with low food females ( $\mathrm{LH})$ generated eggs with 1.4 times higher lipid content than those from low food males that mated with low food females (LL). Low food females that mated with high food males (LH) laid eggs with the highest lipid content compared to all other treatment groups (Figure 2).

When comparing two high food parents (HH) and two low food parents (LL), Multivariate contrasts demonstrated significant parental effects on egg production $(p<0.0001)$. The number of eggs laid contributed the most to this effect followed by egg volume. Two high food parents (HH) laid 1.9 times the number of eggs that two low food parents laid (LL). Additionally, the average volume of eggs produced by two high food parents (HH) was about $4 \%$ larger in volume than eggs laid by two low food parents (LL). When comparing two completely dissimilar parental nutrient mating crosses (HL vs. LH), MANOVA demonstrated significant parental effects on egg parameters measured with egg count contributing most to the effect followed by egg volume and lipid content which contributed similarly. High food females that mated with low food males (HL) laid on average 25.6 more eggs which were 6.9 percent greater in volume than low food females that mated with high food males (LH). However, low food females that mated with high food males (LH) laid eggs with 1.4 times greater lipid content than high food females that mated with low food males (HL) (Figure 2).

\section{DISCUSSION}

Previous research on mosquito Ae. aegypti, found significant parental larval nutrient effects on offspring life history traits (Zirbel et al. 2018, Zirbel and Alto 2018). Results were largely context dependent and sexspecific (Zirbel et al. 2018, Zirbel and Alto 2018) and the potential mechanisms behind these effects were not investigated. Egg size is used as an indicator of parental investment in offspring, and as a measure of parental effects (Uller 2008). Despite this, egg size is not always a reliable indicator of egg quality or composition (Fox and Czesak 2000, McIntyre and Gooding 2000, Giron and Casas 2003). Here we investigated maternal and paternal larval nutrition influences on the number of offspring produced, size, and macronutrient allocation in Ae. aegypti mosquito eggs.

\section{Maternal effects}

We found evidence that maternal larval nutrition influences the number of eggs produced by females as well as their lipid investment. In Diptera, numbers of eggs produced are highly correlated with female body size (Berrigan 1991). It is also argued that a tradeoff exists between egg size and egg number (Smith and Fretwell 1974, Berrigan 1991, Fox and Czesak 2000). In Ae. aegypti larval nutrition strongly influences adult female body size (Schneider et al. 2004) and body size influences the number of offspring produced by females (Steinwascher 1984, Briegel 1990, Dieng et al. 2016). Similarly, there is a general relationship 
between fecundity and body size in most insect taxa (Honĕk 1993). Additionally, Ae. aegypti larval nutrition influences female metabolic reserves, which ultimately influence fecundity for the first ovarian cycle, with high-reserve females producing more eggs than low-reserve females (Briegel 1990, Telang et al. 2006). Previous studies have found that larger females can lay 4-5 times more eggs than smaller females (Briegel 1990). Consistent with these studies, we found that high nutrient, larger females laid twice the number eggs during the first ovarian cycle than low nutrient, smaller females regardless of paternal nutrition. In the current study, the difference between eggs laid by larger versus smaller females was less than Briegel (1990). The discrepancy between our observations and others in the number of eggs produced may be attributable to the range of sizes of adult mosquitoes or the source of host blood (Bennett 1970, Gunathilaka et al. 2017). While we found that high nutrient females laid significantly more eggs than their low nutrient counterparts, we did not assess the viability of the eggs, hatching rates, or subsequent offspring phenotypes and these factors should be considered in future studies.

We also found that when mated with high nutrient males, low nutrient females laid eggs with significantly higher lipid content than those laid by high food females. This is surprising given that high larval nutrient females have been found to be lipid rich relative to low larval food females (Telang et al. 2006) and mating does not appear to alter nutritional state of females in terms of whole body glycogen, lipid, and protein content (Klowden and Chambers 1991). Lipid content in Ae. aegypti eggs is highly variable (Briegel 1990) and so underlying plasticity in lipid resource allocation to eggs may be one source of maternal effects in this system. Previously, we showed that when low nutrient females mated with high nutrient males, subsequent offspring developed more rapidly than other mating treatment groups (Zirbel and Alto 2018). Additional lipids allocated by mothers may, in part, explain the faster development time demonstrated in the previous study.

The availability of maternally-derived lipid reserves is critical during pharate first-instar quiescence, which allows the unhatched larva to survive during unfavorable environmental conditions (Clements 1992, Vinogradova et al. 2007). In unpredictable and variable environments, females may use alternative provisioning strategies to maximize fitness (Olofsson et al. 2009), and increased lipid reserves may improve the ability of eggs to survive quiescence during unfavorable environmental conditions (e.g., dry season and drought). The ability of Ae. aegypti eggs to undergo prolonged quiescence has contributed significantly to the range expansion of this species (Vinogradova 1965, Clements 1992). Also, a laboratory study found that females reared from eggs that have undergone extended quiescence, were more tolerant to starvation and had longer lifespans than those from short quiescence eggs (Perez and Noriega 2013). Other model animals exhibit a positive relationship between starvation resistance and body lipid content (e.g., Drosophila spp., Lee and Jang 2014, Ballard et al. 2008). Increasing lipid content in eggs, may allow for extended pharate larval quiescence, and ultimately greater survival of female offspring. In stressful environments, maternal survival is likely reduced, and females may allocate additional resources to compensate for reduced life span.

\section{Paternal effects}

We found paternal effects on macronutrient allocation in eggs when males mated with low nutrient females but not high nutrient females. Specifically, when high nutrient males mated with low nutrient females, resulting eggs had higher lipid content than when low nutrient males mated with low nutrient females. The transfer of juvenile hormone III in male accessory gland fluid to females during mating has been shown to reduce the likelihood of follicular resorption and increase storage lipids in females (Clifton et al. 2014). This may explain the higher lipid content in eggs from low nutrient females that mated with high nutrient males. Previous study on this system, demonstrated significant paternal effects on offspring life history traits when males mated with high nutrient females but not low nutrient females (Zirbel and Alto 2018) which is different than what we found. Given that environmental stressors can be life-stage specific (Loeschcke and Krebs 1996, De Block and Stoks 2005), it is possible that additional lipid content in eggs buffers against environmental stressors experienced in the egg stage and do not translate to the larval and adult stages. A study conducted by Fernandez and Klowden (1995) found that starved males transferred less protein during 
mating. Male accessory gland proteins in Ae. aegypti modulate host seeking behavior, prevent subsequent mating, and stimulate oviposition (Leahy and Craig 1965, Lee and Klowden 1999). Given that seminal fluid proteins influence female physiology, this may explain why we saw high nutrient males, but not low nutrient males, influencing egg production in low food females. Larger males produce more spermatozoa than smaller males (Ponlawat and Harrington 2007) and this likely applies to seminal fluid proteins. Although previous research suggests that even small quantities of seminal fluid proteins, transferred by small males reared on suboptimal larval nutrients, were enough to render females refractory to subsequent matings (Dickinson and Klowden 1997), it is possible that larger quantities of these proteins may have higher impact on female reproduction than smaller quantities.

\section{Additional comparisons}

When considering two high nutrient parents compared to two low nutrient parents, we found significant effects on egg production with two high nutrient parents laying a higher number of larger eggs than two low nutrient parents. Diet quality often determines size and is correlated with good condition (Blanckenhorn 2000), as such it is expected that two high quality parents will have increased reproductive output. Given the importance of larval nutrition on life history traits in mosquitoes (Teng and Apperson 2000, Arrivillaga and Barrera 2004, Farjana et al. 2012, Couret et al. 2014), it is unsurprising that we would see these effects on reproductive output, especially for the first gonotrophic cycle. Previous studies have shownAe. aegypti females that are nutritionally-stressed as larvae tend to be smaller as adults and lay fewer eggs per clutch (Reyes-Villaneuva 2004, Farjana and Tuno 2013).

Lastly, when considering egg production by high nutrient females mated with low nutrient males (HL) versus low nutrient females mated with high nutrient males (LH), we also found significant effects. Low nutrient females that mated with high nutrient males laid fewer and smaller eggs with higher lipid content than high nutrient females that mated with low nutrient males. Differences in egg quantity between high and low nutrient females was expected given that egg number is correlated with female size in this system (Steinwascher 1984, Briegel 1990, Dieng et al. 2016). However, the increased lipid content was unexpected and as discussed previously may indicate increased resource provisioning in eggs in response to nutrient deprivation.

\section{Conclusions}

Life-history theory predicts that reproductive effort is likely to vary, and that investment in reproduction will be high when expected returns in fitness are high (Brommer 2000). Additionally, mate quality is likely to influence reproductive investment, with higher quality mates leading to greater investment in reproduction (Sheldon 2000). Here, female and male larval nutrition influenced egg quantity and composition in Ae. aegypti. High nutrient females laid the greatest number of eggs, regardless of male nutrition, and eggs produced had similar quantities of proteins and lipids. These results suggest that male quality did not influence female reproductive effort for high nutrient females. It is possible that male quality may influence transfer of other materials to eggs, that were not measured in this study, such as vitamins, minerals, and hormones. Future studies should consider how male quality affects the transfer of additional substances to eggs as well as the viability of eggs produced.

The quantity of lipids in eggs was similar in treatment groups with the exception of eggs laid by low nutrient females that mated with high nutrient males. When low nutrient females mated with high nutrient males, they laid eggs with the highest lipid content relative to all other treatment groups. Lipid content is associated with ability to undergo quiescence and this ability may confer an advantage for stressful conditions encountered by offspring, which may be indicative of an adaptive parental effect. Regardless, additional reproductive effort in eggs produced by low nutrient females that mated with high nutrient males, indicates support for the differential allocation hypothesis (Burley at al. 1988). Differential allocation in this system may only be evident when females experience nutritional deprivation. Females produce larger gametes than males, and consequently the nutritional status of the female may be more important than that of the male. 
Results of this study support differential reproductive investment in offspring but only when females have limited food resources. Results do not support the reproductive compensation hypothesis which predicts that females increase reproductive effort when mating with low quality mates to counteract negative effects on offspring fitness (Gowaty et al. 2007). Larval nutrition influences both female and male quality, and likely that of subsequent offspring.

\section{ACKNOWLEDGEMENTS}

We thank S. Bellamy and S. Ortiz for assistance with the experiments and C.R. Connelly for providing insectary space. We thank C. McKenzie for providing lab space at the USDA Horticultural Research Laboratory and E.M. Branca and M. Clifton for assistance with the macronutrient assays. We thank L.P. Lounibos, C. Smartt, and C. St. Mary for reviewing early versions of the manuscript.

\section{ANIMAL WELFARE}

Chicken care and use was reviewed and approved by the Institutional Animal Care and Use Committee (IACUC Protocol 201807682) of the University of Florida.

\section{REFERENCES}

Agrawal, A.A., C. Laforsch, and R. Tollrian. (1999). Transgenerational induction of defences in animals and plants. Nature . 401: 60-63.

Armbruster, P., and R. Hutchinson. (2002). Pupal mass and wing length as indicators of fecundity in Aedes albopictus and Aedes geniculatus(Diptera: Culicidae). J. Med. Entomol. 39: 699-704.

Arrivillaga, J., and R. Barrera. (2004). Food as a limiting factor for Aedes aegyptiin water-storage containers. J. Vector Ecol. 29: 11-20.

Ballard, J.W., Melvin, R.G., Simpson, S.J. (2008). Starvation resistance is positively

correlated with body lipid proportion in five wild caughtDrosophila simulans populations. J. Insect Physiol. 54(9): 1371-1376.

Bennett, G.F. (1970). The influence of blood meal type on the fecundity of Aedes(Stegomyia) aegypti L. (Diptera: Culcidae). Can. J. Zool.48: 539-543.

Bernardo, J. (1996). The particular maternal effect of propagule size, especially egg size: patterns, models, quality of evidence and interpretations. Am. Zool. 36: 216-236.

Berrigan, D. (1991). The allometry of egg size and number in insects. Oikos. 60: 313-321.

Blanckenhorn, W.U. (2000). The evolution of body size: what keeps organisms small? Q. Rev. Biol. 75: 385-407.

Bonduriansky, R., and M.H. Head. (2007). Maternal and paternal condition effects on offspring phenotype in Telostylinus angusticollis (Diptera: Neriidae).J. Evol. Biol. 20: 2379-2388.

Bongiorni, S., O. Cintio, and G. Prantera. (1999). The relationship between DNA methylation and chromosome imprinting in the Coccid Planococcus citri .Genetics. 151: 1471-1478.

Boots, M., and K.E. Roberts. (2012). Maternal effects in disease resistance: poor maternal environment increases offspring resistance to an insect virus.Proc. R. Soc. B. 279: 4009-4014.

Briegel, H. (1990). Metabolic relationship between female body size, reserves, and fecundity of Aedes aegypti . J. Insect Physiol. 36: 165-172.

Briegel, H., T. Gut, and A.O. Lea. (2003). Sequential deposition of yolk components during oogenesis in an insect, Aedes aegypti (Diptera: Culicidae).J. Insect. Physiol. 49: 249-260.

Brommer, J.E. (2000). The evolution of fitness in life-history theory.Biol. Rev. 75: 377-404. 
Burley, N. (1988). The differential allocation hypothesis: An experimental test. Am. Nat. 123(5):611-628.

Carrière, Y. (1994). Evolution of phenotypic variance: non-Mendelian parental influences on phenotypic and genotypic components of life-history traits in a generalist herbivore. Heredity. 72: 420-430.

Clements, A.N. (1992). The biology of mosquitoes: Vol. I. Chapman and Hall, London, UK.

Clifton, M.E., S. Correa, C. Rivera-Perez, M. Nouzova, and F.G. Noriega. (2014). Male Aedes aegypti mosquitoes use JH III transferred during copulation to influence previtellogenic ovary physiology and affect the reproductive output of female mosquitoes. J. Insect Physiol. 64: 40-47.

Couret, J.E., E. Dotson, and M.Q. Benedict. (2014). Temperature, larval diet, and density effects on development rate and survival of Aedes aegypti (Diptera: Culicidae). PLoS One. 9: e87468. DOI: 10.1371/journal.pone.0087468.

De Block, M., and R. Stoks. (2005). Fitness effects from egg to reproduction: Bridging the life history transition. Ecology. 86: 185-197.

Dew-Budd, K., J. Jarnigan, and L.K. Reed. (2016). Genetic and sex-specific transgenerational effects of a high fat diet in Drosophila melanogaster. PLoS One. 1: e0160857. DOI: 10.1371/journal.pone.0160857.

Dickinson, J.M. and M.J. Klowden. (1997). Reduced transfer of male accessory gland proteins and monadry in female Aedes aegypti mosquitoes. J. Vector Ecol. 22: 95-98.

Farjana, T., and N. Tuno. (2013). Multiple blood feeding and host-seeking behavior in Aedes aegypti and Aedes albopictus (Diptera: Culicidae). J. Med. Entomol. 50: 838-846.

Farjana, T., N. Tuno, and Y. Higa. (2012). Effects of temperature and diet on development and interspecies competition in Aedes aegypti and Aedes albopictus . Med. Vet. Entomol. 26: 210-217.

Fernandez, N.M., and M.J. Klowden. (1995). Male accessory gland substances modify the host-seeking behavior of gravid Aedes aegypti mosquitoes.J. Insect Physiol. 41: 965-970.

Fox, C.W., and M.E. Czesak. (2000). Evolutionary ecology of progeny size in arthropods. Annu. Rev. Entomol. 45: 341-369.

Fox, C.W., and T.A. Mousseau. (1996). Larval host plant affects fitness consequences of egg size variation in the seed beetle Stator limbatus . Oecologia.107: 541-548.

Fox, C.W., M.E. Czesak, T.A. Mousseau, and D.A. Roff. (1999). The evolutionary genetics of an adaptive maternal effect: Egg size plasticity in a seed beetle.Evolution. 53: 552-560.

Freitak, D., D.G. Heckel, and H. Vogel. (2009). Dietary-dependent transgenerational immune priming in an insect herbivore. Proc. R. Soc. B. 276: 2617-2624.

Fuchs, M.S., Craig, Jr., G.B., Despommier, D.D. (1969). The protein nature of the substance inducing female monogamy in Aedes aegypti. J. Insect Physiol. 15(4): 701-709.

Garcia-Gonzalez, F., and L.W. Simmons. (2011). Good genes and sexual selection in dung beetles (Onthophagus taurus ): Genetic variance in egg-to-adult and adult viability. PLoS One. 6: e16233. DOI: 10.1371/journal.pone.0016233.

Gibbs, M., C.J. Breuker, H. Hesketh, R.S. Hails, and H. Van Dyck. (2010). Maternal effects, flight versus fecundity trade-offs, and offspring immune defence in the Speckled Wood butterfly, Pararge aegeria . BMC Evol. Biol.10: 345-355.

Giron, D. and J. Casas. (2003). Mothers reduce egg provisioning with age. Ecol. Lett. 6: 273-277.

Giron, D., A. Rivero, N. Mandon, E. Darrouzet, and J. Casas. (2002). The physiology of host feeding in parasitic wasps: implications for survival. Funct. Ecol. 16: 750-757. 
Gowaty, P.A., W.W. Anderson, C.K. Bluhm, L.C. Drickamer, Y.K. Kim, and A.J. Moore. (2007). The hypothesis of reproductive compensation and its assumptions about mate preferences and offspring viability. Proc. Natl. Acad. Sci. USA.104: 15023-15027.

Gunathilaka, N., T. Ranathunge, L. Udayanga, and W. Abeyewickreme. (2017). Efficacy of blood sources and artificial blood feeding methods in rearing of Aedes aegypti (Diptera: Culicidae) for sterile insect technique and incompatible insect technique approaches in Sri Lanka. BioMed Res. Int. 3196924. DOI: $10.1155 / 2017 / 3196924$.

Hawley, W.A. (1985). A high-fecundity Aedine: Factors affecting egg production of the Western treehole mosquito, Aedes sierrensis (Diptera: Culicidae).J. Med. Entomol. 22: 220-225.

Helinski, M.E.H. and L.C. Harrington. (2011). Male mating history and body size influence female fecundity and longevity of the dengue vector Aedes aegypti. J. Med. Entomol. 48: 202-211.

Honĕk, A. (1993). Intraspecific variation in body size and fecundity in insects: a general relationship. Oikos . 66: 483-492.

Kirkpatrick, M., and R. Lande. (1989). The evolution of maternal characters. Evolution.43: 485-503.

Klowden, M.J., and G.M. Chambers. (1991). Male accessory gland substances activate egg development in nutritionally stressed Aedes aegypti mosquitoes.J. Insect Physiol. 37: 721-726.

Lee K.P., Jang, T. (2014). Exploring the nutritional basis of starvation resistance in

Drosophila melanogaster . Funct. Ecol. 28:1144-1155.

Loeschcke, V., and R.A. Krebs. (1996). Selection for heat-resistance in larval and in adult Drosophila buzzatii : Evolution. 50: 2354-2359.

MacDonald, W.A. (2012). Epigenetic mechanisms of genomic imprinting: common themes in the regulation of imprinted regions in mammals, plants, and insects.Genet. Res. Int. DOI: 10.1155/2012/585024

Marshall, D.J., and T. Uller. (2007). When is a maternal effect adaptive? Oikos. 116: 1957-1963.

McIntyre, G.S., and R.H. Gooding. (2000). Egg size, contents, and quality: maternal-age and -size effects on house fly eggs. Can. J. Zool. 78: 1544-1551.

Moran, N.A., J.P. McCutcheon, and A. Nakabachi. (2008). Genomics and evolution of heritable bacterial symbionts. Annu. Rev. Genet. 42: 165-90.

Mousseau, T.A., and H. Dingle. (1991). Maternal effects in insect life histories. Annu. Rev. Entomol. 36: 511-534.

Mousseau, T.A., and C.W. Fox. (1998). Maternal effects as adaptations. Oxford University Press, New York, NY.

Olofsson, H., J. Ripa, and N. Jonzén. (2009). Bet-hedging as an evolutionary game: the trade-off between egg size and number. Proc. R. Soc. B. 276: 2963-2969.

Perez, M.H., and F.G. Noriega. (2012). Aedes aegypti pharate 1st instar quiescence affects larval fitness and metal tolerance. J. Insect Physiol.58: 824-829.

Perez, M.H., and F.G. Noriega. (2013). Aedes aegypti pharate 1st instar quiescence: A case for anticipatory reproductive plasticity. J. Insect Physiol.59: 318-324.

Plaistow, S.J., C.T. Lapsley, and T.G. Benton. (2006). Context-dependent intergenerational effects: the interaction between past and present environments and its effect on population dynamics. Am. Nat. 167: 206-215. 
Pölkki, M., K. Kangassalo, and M.J. Rantala. (2012). Transgenerational effects of heavy metal pollution on immune defense of the blow fly Protophormia terraenovae. PLoS One. 7: e38832. DOI: 10.1371/journal.pone.0038832.

Ponlawat, A., and L.C. Harrington. (2007). Age and body size influence male sperm capacity of the dengue vector Aedes aegypti (Diptera: Culicidae). J. Med. Entomol. 44: 422-427.

Qvarnström, A., and T.D. Price. (2001). Maternal effects, paternal effects and sexual selection. Trends Ecol. Evol. 16: 95-100.

Reyes-Villaneuva, F. (2004). Egg development may require multiple bloodmeals among smallAedes aegypti (Diptera: Culicidae) field collected in northeastern Mexico. Fla. Entomol. 87: 630-632.

Rolff, J. (1999). Parasitism increases offspring size in a damselfly: experimental evidence for parasite-mediated maternal effects. Anim. Behav. 58: 1105-1108.

Rossiter, M.C. (1991). Environmentally-based maternal effects: a hidden force in insect population dynamics? Oecologia. 87: 288-294.

Roth, O., G. Joop, J. Eggert, J. Hilbert, J. Daniel, P. Schmid-Hempel, and J. Kurtz. (2009). Paternally derived immune priming for offspring in the red flour beetle, Tribolium castaneum. J. Anim. Ecol. 79: 403413.

Scheiner, S.M. (2001). MANOVA: multiple response variables and multispecies interactions, pp. 99-115. In: S.M. Scheiner and J. Gurevitch (eds.), Design and Analysis of Ecological Experiments. Oxford University Press; Oxford, UK.

Schneider, J.R., A.C. Morrison, H. Astete, T.W. Scott, and M.L. Wilson. (2004). Adult size and distribution of Aedes aegypti (Diptera: Culicidae) associated with larval habitats in Iquitos, Peru. J. Med. Entomol. 41: 634-642.

Sheldon, B.C. (2000). Differential resource allocation: tests, mechanisms and implications. Trends Ecol. Evol. 15: 397-402.

Simmons, L.W., Lovegrove, M. (2019). Nongenetic paternal effects via seminal

fluid. Evol. Lett. 3(4): 403-411.

Sinervo, B. (1990). The evolution of maternal investment in lizards: an experimental and comparative analysis of egg size and its effects on offspring performance.Evolution. 44: 279-294.

Sinervo, B. (1991). Experimental and comparative analyses of egg size in lizards: constraints on the adaptive evolution of maternal investment per offspring, pp. 725-734. In: E.C. Dudley (ed.), The unity of evolutionary biology. Dioscorides Press; Portland, OR.

Sinervo, B. and P. Licht. (1991). Proximate constraints on the evolution of egg size, number, and total clutch mass in lizards. Science. 252: 1300-1302.

Sirot, L.K., M.C. Hardstone, M.E.H. Helinksi, J.M.C. Ribeiro, M. Kimura, P. Deewatthanawong, M.F. Wolfner, and L.C. Harrington. (2011). Towards a semen proteome of the dengue vector mosquito: Protein identification and potential functions. PLoS Neglected Trop. Dis. 5: e989.

Smith, C.C., and S.D. Fretwell. (1974). The optimal balance between size and number of offspring. Am. Nat. 108: 499-506.

Steinwascher, K. (1984). Egg size variation in Aedes aegypti : relationship to body size and other variables. Am. Midl. Nat. 112: 76-84.

Stearns, S. (1992). The evolution of life histories. Oxford University Press, London xii, 249 pp.

Telang. A., Y. Li, F.G. Noriega, and M.R. Brown. (2006). Effects of larval nutrition on the endocrinology of mosquito egg development. J. Exp. Biol. 209: 645-655. 
Teng, H.J., and C.S. Apperson. (2000). Development and survival of immature Aedes albopictus and Aedes triseriatus (Diptera: Culicidae) in the laboratory: effects of density, food, and competition on response to temperature. J. Med. Entomol. 37: 40-52.

Tidbury, H.J., A.B. Pedersen, and M. Boots. (2010). Within and transgenerational immune priming in an insect to a DNA virus. Proc. R. Soc. B. 278: 871-876.

Triggs, A.M., and R.J. Knell. (2012). Parental diet has strong transgenerational effects on offspring immunity. Funct. Ecol. 26: 1409-1417.

Uller, T. (2008). Developmental plasticity and the evolution of parental effects. Trends Ecol. Evol. 23: 432438.

Urbanski, J.M., J.B. Benoit, M.R. Michaud, D.L. Denlinger, and P. Armbruster. (2010). The molecular physiology of increased egg desiccation resistance during diapause in the invasive mosquito, Aedes albopictus . Proc. R. Soc. B. 7: 2683-2692.

Vijendravarma, R.K., S. Narasimha, and T.J. Kawecki. (2010). Effects of parental diet on egg size and offspring traits in Drosophila. Biol. Lett. 6: 238-241.

Vinogradova, E., V. Alekseev, B. Stasio, and J. Gilbert. (2007). Diapause in aquatic insects, with emphasis on mosquitoes, pp. 83-113. In: H.J. Dumont and M.J.A. Werger (eds.), Diapause in aquatic invertebrates: theory and human use. Springer; Dordrecht, NLD.

Winkler, D.W., and K. Wallin. (1987). Offspring size and number: A life history model linking effort per offspring and total effort. Am. Nat. 129: 708-720.

Zanchi, C., J.P. Troussard, G. Martinaud, J. Moreau, and Y. Moret. (2011). Differential expression and costs between maternally and paternally derived immune priming for offspring in an insect. J. Anim. Ecol. 80: 1174-1183.

Ziegler, R. (1997). Lipid synthesis by ovaries and fat body of Aedes aegypti (Diptera: Culicidae). Eur. J. Entomol. 94: 385-391.

Zirbel, K., B. Eastmond, and B.W. Alto. (2018). Parental and offspring larval diets

interact to influence life history traits and dengue virus infection of offspring in Aedes aegypti. R. Soc. Open Sci. 5(7): 180539.

Zirbel, K., and B.W. Alto. (2018). Maternal and paternal nutrition in a mosquito

influences offspring life histories but not infection with an arbovirus.Ecosphere 9(10): 10.1002/ecs2.2469.

FIGURES AND TABLES

Table 1. Summary statistics for parental treatment groups

\begin{tabular}{llllll}
\hline Treatment & $\#$ & $\#$ & \% Blood fed (n) & \% Laid eggs $(\mathrm{n})$ & Blood fed Wing Length $(\mathrm{mm}) \pm$ SE \\
\hline HH & 207 & 207 & $59.9(124)$ & $81.5(101)$ & $2.45 \pm 0.02$ \\
HL & 207 & 207 & $77.8(161)$ & $81.4(131)$ & $2.45 \pm 0.01$ \\
LH & 248 & 248 & $38.7(96)$ & $78.1(75)$ & $2.07 \pm 0.02$ \\
LL & 248 & 248 & $51.2(127)$ & $70.9(90)$ & $2.09 \pm 0.01$ \\
\hline
\end{tabular}

Table 2. MANOVA for treatment effects and multivariate pairwise contracts of parental larval nutrient mating cross on egg count, egg volume $\left(\mathrm{mm}^{3}\right)$, lipid content ( $\left.\mathrm{pmol} / \mathrm{egg}\right)$, and protein content ( $\left.\mu \mathrm{g} / \mathrm{egg}\right)$. 


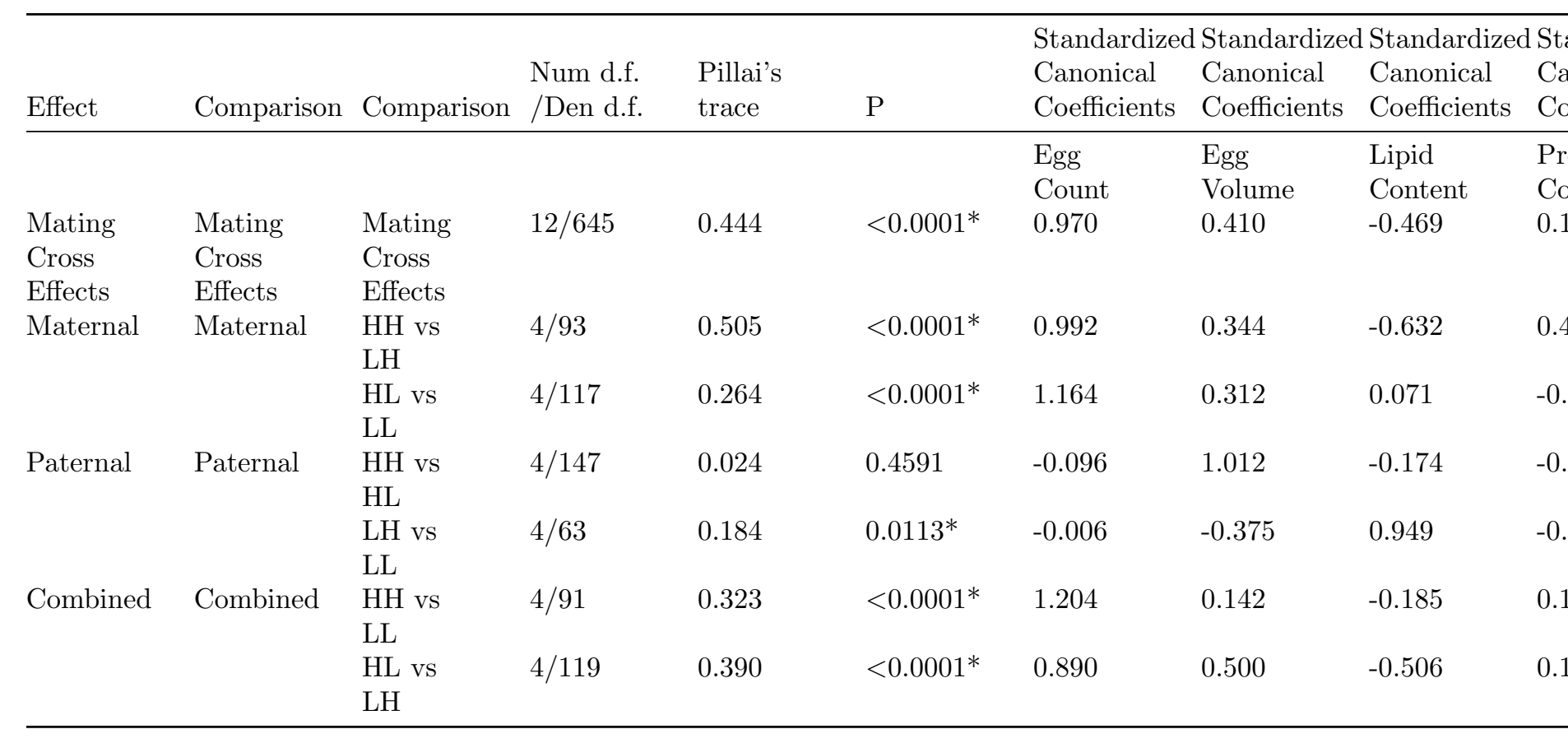

*Denotes significant p-value after Bonferroni correction 


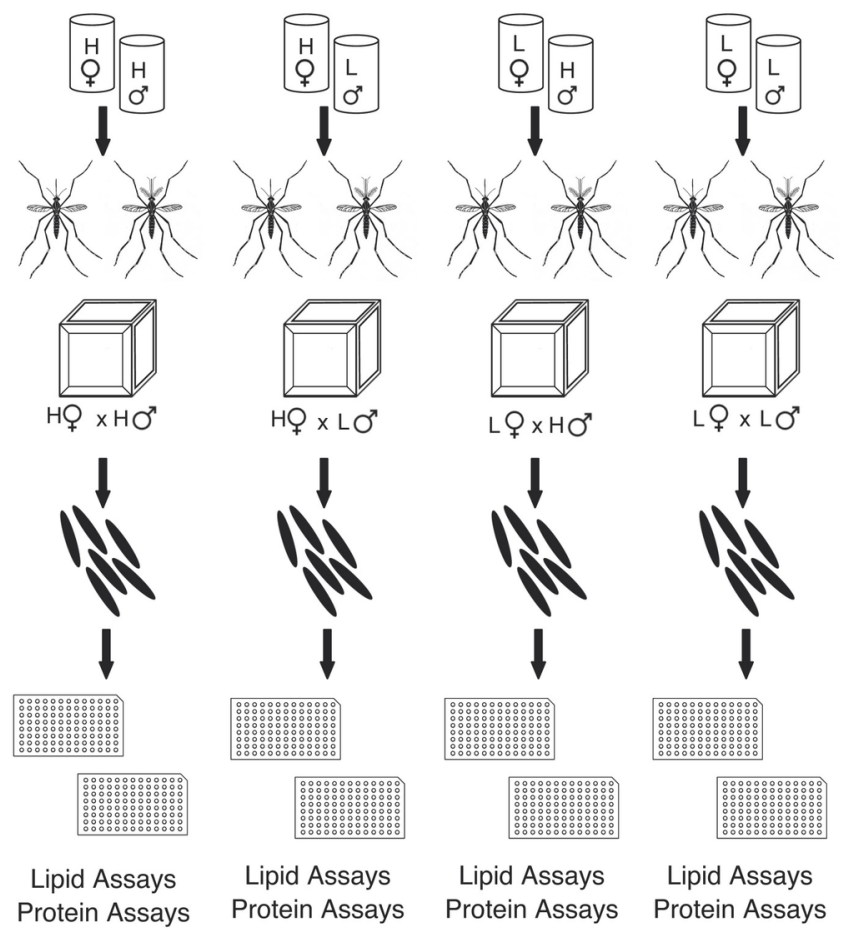

Figure 1. Experimental design describing parental mating crosses and assays. 

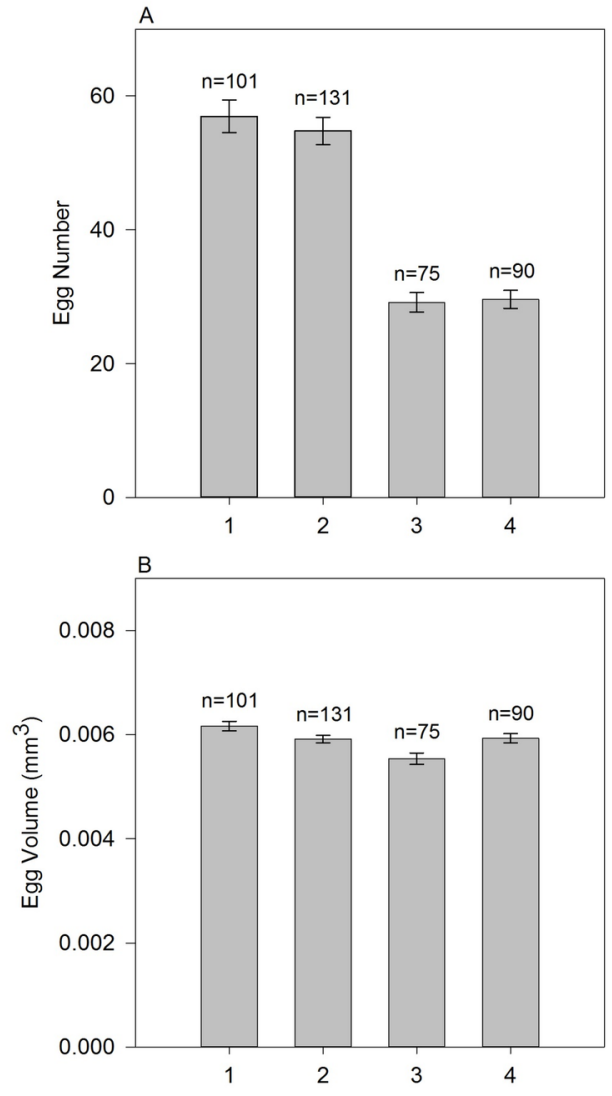

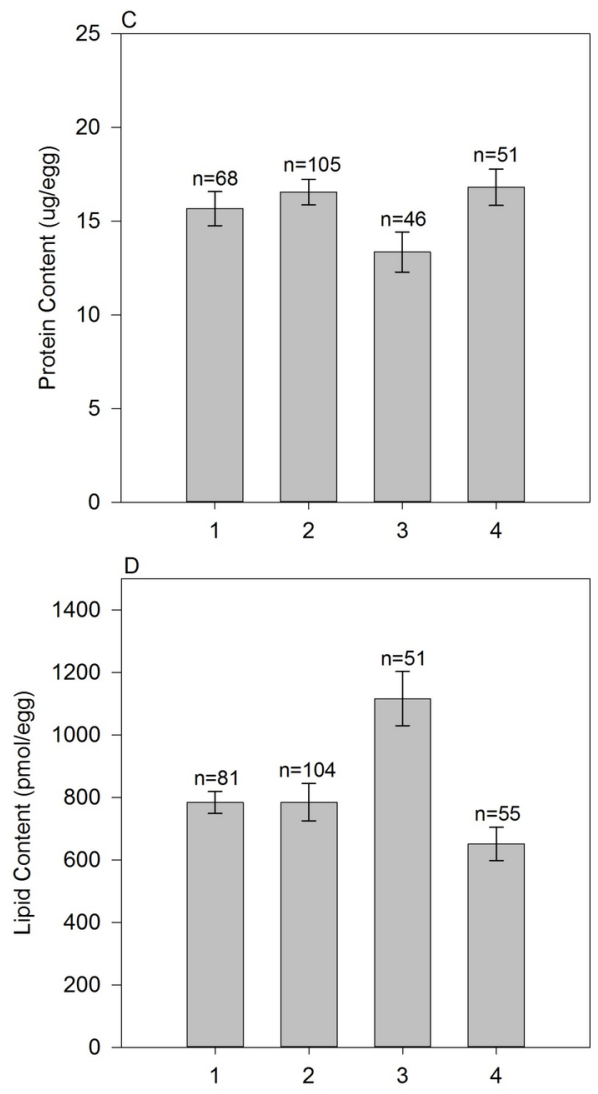

Figure 2. Mean descriptive statistics ( \pm S.E.) by parental treatment group with $1=\mathrm{HH}, 2:=\mathrm{HL}, 3$ : LH, and 4: LL for A) Egg Count, B) Egg Volume, C) Protein Content and D) Lipid Content.

Figure 2. Continued 